Radial and Nonradial Pulsations as Probes of Stellar Physics

ASP Conference Series, Vol. 259, 2002

C. Aerts, T.R. Bedding, \& J. Christensen-Dalsgaard, eds.

\title{
WWW Archive of Fourier Coefficients
}

\section{S. Morgan}

Department of Earth Science, University of Northern Iowa, Cedar Falls Iowa, USA

\begin{abstract}
Fourier coefficients are a valuable tool in the study of a wide variety of pulsating stars. They can be used to derive various physical parameters, including mass, luminosity, metallicity and effective temperature and are frequently used to discriminate between different pulsation modes. With the increase in large-scale surveys and the availability of data on the Internet, the number of Fourier coefficients available for study has expanded greatly and it is difficult to find all current data for individual stars or a subset of stars. To assist others in obtaining and making use of Fourier coefficients, an archive of published values of Fourier coefficients has been set up. Users can search for data on individual stars or for a range of parameters. Several Java programs are used to display the data in a variety of ways. The archive is located at the Web site http://www.earth.uni.edu/fourier/.
\end{abstract}

\section{Database information}

The Fourier Coefficient database is an attempt to place all currently available data in a central location, as well as providing bibliographical references for researchers. This includes all data concerning intrinsic variables such as $R R$ Lyraes, Cepheids, SX Phe stars, $\delta$ Scuti stars and Mira variables. Wherever possible, the original data are provided, though for meaningful comparisons (such as graphs), the $\phi_{i j}$ values have been transformed to the common system that was first defined by Simon \& Lee (1981). The database provides information for each star including period, Right Ascension and declination from the General Catalog of Variable Stars (Kholopov 1985-88), coefficients $A_{0}-A_{8}$ and $\phi_{1}-\phi_{8}$ along with their errors, values of $R_{i j}$ and $\phi_{i j}$ along with their errors, and full bibliographic citations. Wherever possible, an interactive light/velocity curve graph is provided to see how the different Fourier fits compare. In those cases where insufficient data for a curve are provided, the values are not graphed and this is noted in the table containing the data.

Data are sorted in several ways. For galactic (non-globular cluster) variables, individual stars are sorted by constellation and variable type. Type is generally defined by that given in the GCVS, but where this is in error (usually for stars listed as eclipsing variables in the $G C V S$ ), a correction is made. For those stars that have uncertain type, most often short period $\delta$ Scuti, c-type RR Lyrae or SX Phe stars, the GCVS type is usually used, though it is possible that some errors have slipped through the cracks. For variables in clusters, the 
stars are sorted by cluster name, and then are accessible on the individual cluster pages. Data for variable stars in clusters are based on the Clement on-line database (1997) of the Helen Sawyer Hogg Catalogue of Variable Stars in Globular Clusters. At the present time, the cluster data are not yet available, but should be in a short time.

Information about the variables can also be obtained using several search programs. One is a standard search page that allows the user to select stars according to several criteria, including name (or part of a name), period range, type of variable and type of measurement. Results of the $R_{i j}$ and $\phi_{i j}$ values are provided in both tabular and graphical form. Users can also use an interactive $R_{21}$ or $\phi_{21}$ graph to select individual stars from the graphs. Several graphs are also available for downloading, including those with all $R_{i j}$ and $\phi_{i j}$ values plotted versus period.

\section{Future Plans}

At the present time, only galactic Fourier coefficients are available. The globular cluster data are currently being added to the data base, and it is hoped that extragalactic coefficients will be added soon, as will data from some of the recent large-scale surveys. Data from theoretical modeling (hydrodynamic or one-zone models) of phenomena may also be of interest and will be included. It may be advantageous to expand the methods of obtaining data from the database, as well as methods of producing graphs according to users' specifications. Anyone who is interested in contributing to the database or who has suggestions to help improve it are invited to contact the author.

Acknowledgments. This work would have been impossible without several vital Internet resources, including the NASA Astrophysics Data System Abstract Service, the Strasbourg Astronomical Data Center, the Simbad service, the Sternberg Astronomical Institute and Christine Clement's on-line Catalogue of Variable Stars in Globular Clusters. The American Astronomical Society is also thanked for providing funds to travel to this conference.

\section{References}

Clement, C. 1997, available at http://www.astro.utoronto.ca/ cclement/

Kholopov, P. N., et al. 1985-88, General Catalogue of Variable Stars, 4th edition, Volumes I-III, (Moscow, Nauka Publishing House)

Simon, N. R. \& Lee, A. S. 1981, ApJ, 248, 291 